\title{
Vídeos educativos sobre aleitamento materno: educação em saúde online
}

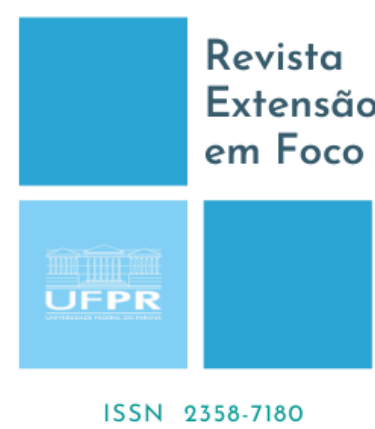

\author{
Rafaeli Musial Scorupski ${ }^{1}$, Luciana Julek ${ }^{2}$, Ana Paula Xavier Ravelli ${ }^{3}$, Murilo Rossi de \\ Matos $^{4}$, Suellen Vienscoski Skupien ${ }^{5}$, Fabiana Bucholdz Teixeira Alves ${ }^{6}$
}

\begin{abstract}
RESUMO
O aleitamento materno é uma das principais práticas de promoção à saúde, a qual proporciona inúmeros benefícios para mãe e filho, atuando principalmente na redução da morbimortalidade infantil. Todo conhecimento acerca do aleitamento materno tem como ferramenta facilitadora a educação em saúde, no qual a informação chega à população por meio dos profissionais de saúde, visando reduzir o desmame precoce. O enfermeiro é um dos profissionais responsáveis pela educação em saúde, ainda, a Tecnologia de Informação e Comunicação (TIC) pode ser uma ferramenta facilitadora do ensino-aprendizagem. Objetivou estruturar vídeos educativos sobre Aleitamento Materno utilizando recursos das tecnologias e aplicar instrumento de avaliação segundo critérios de Pasqualiperante à funcionalidade dos vídeos educativos acerca da temática abordada. Estudo descritivo, de abordagem quantitativa e social. A coleta de dados aconteceu com 4 profissionais especialistas em Saúde da Mulher e 38 acadêmicos do curso de Bacharelado em Enfermagem da $1^{\mathrm{a}}, 2^{\mathrm{a}}$ e $3^{\mathrm{a}}$ séries pela Universidade Estadual de Ponta Grossa, nos meses de março e abril de 2018, a partir de questionários estruturais visando a avaliação preliminar dos vídeos educativos inseridos no website YouTube ${ }^{\circledR}$ e divulgados por meio do Facebook ${ }^{\circledR}$, tendo se apropriado de Pasquali e Escala de Likert. A análise dos dados foi desenvolvida pela estatística descritiva, a partir de frequência simples. Os resultados foram satisfatórios, atingindo $\geq 60 \%$ acertos evidenciando que a ferramenta educativa facilita o processo ensino-aprendizagem.
\end{abstract}

Palavras-chave:Aleitamento Materno. Educação em Saúde.Enfermagem. Tecnologia de Informação.

\section{ABSTRACT}

Breastfeeding is one of the main practices that promote health by providing innumerable benefits for a mother and her child, especially in reducing infant morbidity and mortality. Knowledge about breastfeeding serves as a facilitative tool in health education, in which information reaches the population by means of health professionals, aiming to reduce early weaning. Nurses are one of the many types of health professionals responsible for health education, in conjunction with other teaching-learning tools, such as the Information and Communication Technology (ICT). The objective was to structure educational videos on breastfeeding using technological resources and apply an evaluation tool according

\footnotetext{
${ }^{1}$ Enfermeira. Universidade Estadual de Ponta Grossa (UEPG), Ponta Grossa, Paraná, Brasil. Email:rmscorupski@ hotmail.com. Orcid: https://orcid.org/0000-0002-9451-1180

${ }^{2}$ Enfermeira. Universidade Estadual de Ponta Grossa (UEPG), Ponta Grossa, Paraná, BrasilEmail:lucianajuleka@ @otmail.com.Orcid: https://orcid.org/0000-0001-6898-6839

${ }^{3}$ Doutora em Enfermagem.Universidade Estadual de Ponta Grossa (UEPG), Ponta Grossa, Paraná, Brasil. E-mail:anapxr@hotmail.com. Orcid: https://orcid.org/0000-0003-4095-758X

${ }^{4}$ Enfermeiro. Universidade Estadual de Ponta Grossa (UEPG), Ponta Grossa, Paraná, Brasil. Email:murilorossdematos@yahoo.com.br. Orcid: https://orcid.org/0000-0003-2726-026X

${ }^{5}$ Mestre em Tecnologia em Saúde.Universidade Estadual de Ponta Grossa (UEPG), Ponta Grossa, Paraná, Brasil. E-mail:suvienscoski@ hotmail.com. Orcid: https://orcid.org/0000-0002-9503-6334

${ }^{6}$ Doutora em Odontopediatria.Universidade Estadual de Ponta Grossa (UEPG), Ponta Grossa, Paraná, Brasil. E-mail:fabi.bucholdz@hotmail.com. Orcid:https://orcid.org/0000-0001-9955-1811
} 
to Pasquali's criteria regarding the functionality of the educational videos about the subject matter. This is a descriptive study, with a quantitative and social approach. The data collection was performed by 4 professionals specialized in Women's Health and 38, 1st, 2nd and 3rd year students in the bachelor's nursing program of Ponta Grossa State University. Data was collected in the months of March and April of 2018, from structural questionnaires aiming at the preliminary evaluation of the educational videos uploaded in the website YouTube $®$ and shared throughout Facebook $®$, having appropriated Pasquali and Likert Scale. The analysis of the data was developed by descriptive statistics with simple frequency. The results were satisfactory, reaching $\geq 60 \%$ correctness, showing that the educational tool facilitates the teaching-learning process.

Keywords:Breastfeeding. Health Education. Nursing. Information Technology.

\section{INTRODUÇÃO}

O aleitamento materno é uma das principais práticas de promoção à saúde, a qual proporciona inúmeros benefícios para o binômio mãe e filho, atuando principalmente na redução da morbimortalidade infantil. Reflete no sistema fisiológico da criança, e também contribui para a recuperação da mãe no período puerperal(BRASIL, 2015).

Assim, a prática do aleitamento contribui para um melhor desenvolvimento cognitivo da criança, evitando aparecimento de diarreia, infecção respiratória, diminuindo o risco para alergias, hipertensão, diabetes, colesterol alto e também para obesidade (BRASIL, 2015). Cabe ressaltar que para a puérpera, tal prática ajuda principalmente na recuperação pós-parto, onde os níveis de ocitocina elevam-se, auxiliando a involução uterina e prevenindo hemorragias (AZEVEDO et al., 2015).

Nesse contexto, a Organização Mundial da Saúde (OMS), o Fundo das Nações Unidas para a Infância (Unicef) e o Ministério da Saúde (MS) recomendam o aleitamento materno exclusivo durante os primeiros seis meses de idade, e complementado até os dois anos ou mais (BRASIL, 2015).

Diante do cenário já exposto, todo conhecimento acerca do aleitamento materno tem como ferramenta facilitadora a educação em saúde, no qual a informação chega à população por meio dos profissionais de saúde, visando reduzir o desmame precoce. Entre os profissionais, o enfermeiro se destaca nas esferas primária, secundária e terciária por seu maior envolvimento em todo ciclo gravídico-puerperal da mulher, exercendo assim, um importante papel como educador para saúde (ALVES; OLIVEIRA; RITO, 2018).

Desse modo, cabe ao enfermeiro o incentivo e encorajamento a prática da amamentação, abordando seus benefícios, diagnosticando e intervindo nas dificuldades encontradas pelo binômio mãe/filho nesse processo. Vale ressaltar que, a mulher deve 
ser orientada desde o acompanhamento pré-natal, para que seu conhecimento reflita no momento após o parto e na adesão do ato de amamentar(BAPTISTA et al., 2015).

Nesta perspectiva, há diversas políticas públicas visando aumentar as taxas de amamentação, como o Programa Nacional de Incentivo ao Aleitamento Materno, Iniciativa Hospital Amigo da Criança, Bancos de Leite Humano, Estratégia Amamenta e Alimenta Brasil. Assim, as mesmas estão interligadas com as intervenções comunitárias, na Atenção Primária, Secundária e Terciária, com as visitas domiciliares por profissionais de saúde, aconselhamento, apoio e participação na prática da amamentação (VENANCIO et al., 2016).

Destaca-se que a educação em saúde permeia em todas as etapas do ciclo gravídico-puerperal, visto que colabora para a promoção de saúde e qualidade de vida do binômio mãe/filho. Cabe ao enfermeiro desenvolver ações que possibilitem a autonomia da parturiente em relação ao pré-natal, parto, cuidados com o bebê, puerpério e aleitamento materno (SILVA et al., 2017).

Para o desenvolvimento das práticas educativas podem ser utilizadas diversas ações de promoção de saúde, como as orientações realizadas no grupo de gestantes, na sala de espera, as visitas domiciliares, entre outros. Como estratégias facilitadoras do processo ensino-aprendizagem também se destacam as inovações tecnológicas, pois podem ser utilizadas como uma forma dinâmica de dissipar o conhecimento, o que desperta um maior interesse na aprendizagem (SILVA et al., 2016).

Com o objetivo de incentivar à prática do aleitamento materno, várias tecnologias têm sido utilizadas como ferramentas para facilitar o aprendizado das puérperas. Um estudo realizado identificou tecnologias que facilitam o processo de aprendizagem, como o álbum seriado, manual educativo, tele amamentação, CD-ROM, vídeo conferência e literatura de cordel. (SILVA et al., 2016).

Salienta-se que a Tecnologia de Informação e Comunicação (TIC) permite uma expansão do ensino e da aprendizagem, pois amplia o ensino, sendo uma aliada no processo de inclusão social (MARTINS, 2013). Assim, as tecnologias educativas têm como objetivo auxiliar no processo ensino-aprendizagem e a realizar práticas educativas na própria comunidade ou com determinados sujeitos (ALVES; OLIVEIRA; RITO, 2018). 
Desta forma, neste estudo a questão norteadora é que o uso da TIC (Web 2.0) contribui para promover educação em saúde facilitando o ensino-aprendizado frente ao tema aleitamento materno, utilizando a internet e suas ferramentas. A TIC reflete na área de saúde, pois pode ser utilizada como um meio para tratar a informação e dissipála na rede, contribuindo para a promoção do cuidado via online (SANTOS, 2017).

Portanto, visando realizar a educação em saúde de maneira atrativa e diferenciada, este estudo objetivou estruturar vídeos educativos sobre Aleitamento Materno utilizando recursos das TIC e aplicar instrumento de avaliação segundo critérios de Pasqualiperante à funcionalidade dos vídeos educativos acerca da temática abordada. Os objetivos específicos foram: criar website no YouTube ${ }^{\circledR}$ para armazenar os vídeos educativos sobre aleitamento materno e compartilhar e divulgá-los em página do Projeto CEPP (Consulta de Enfermagem no Pré-natal e Pós-parto) no website Facebook® para toda a população com acesso à internet.

\section{MÉTODOS}

Estudo descritivo e de abordagem quantitativa e social. A coleta de dados foi realizada com 42 participantes, sendo-os: 4 enfermeiras especialistas em Saúde da Mulher/Obstetrícia, 38 alunos do curso de Bacharelado em Enfermagem da Universidade Estadual de Ponta Grossa PR, sendo 8 alunos da primeira série, 15 alunos da segunda série e 15 alunos da terceira série, nos meses de março e abril de 2018.

Este estudo tem como âncora o projeto Consulta de Enfermagem no Pré-Natal e Pós-parto que desenvolve ações de educação em saúde no período puerperal e aleitamento materno. A consulta de enfermagem envolve a participação de acadêmicos do curso de Bacharelado em Enfermagem da $4^{\circ}$ e $5^{\circ}$ série, os quais esclarecem dúvidas maternas numa linguagem clara e acessível, que neste estudo se apropriou das TIC visando a educação em saúde online. Cabe ressaltar que o referido projeto possui página em rede social Facebook ${ }^{\circledR}$ (Figura 1) disponível no endereço eletrônico https://www.facebook.com/ceppuepg. 
Figura 1 - Projeto CEPP no website Facebook ${ }^{\circledR}$

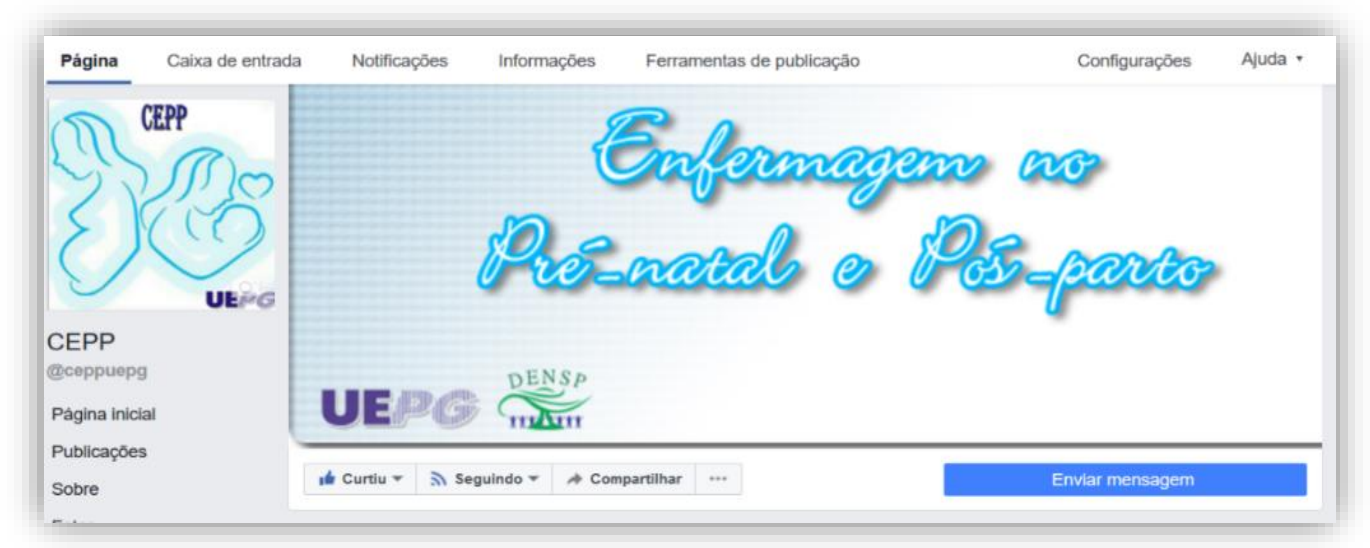

Fonte: autores (2018).

A elaboração e edição dos vídeos foi resultado de uma parceria com o Núcleo de Tecnologia e Educação Aberta e a Distância (NUTEAD) da Universidade Estadual de Ponta Grossa. As tecnologias de informação utilizadas no ensino à distância são gerenciadas pelo Núcleo de Vídeos e Conferências (NUVC), o qual possui estúdios para as gravações das videoaulas, equipamentos inovadores como o teleprompter, som e luminosidade adequada.

Foram elaborados 5 vídeos de duração aproximada de 3 minutos cada. Os mesmos são compostos por assuntos sobre aleitamento materno, com recomendações baseadas no Ministério da Saúde As temáticas trabalhadas foram: aleitamento materno exclusivo (benefícios para mãe e filho, definições de aleitamento materno, recomendações), tipos de leite (colostro, leite de transição, leite maduro, diferenças na composição do leite), pega correta (posicionamento correto, pega adequada e má pega), fissura mamária (definição, porque ocorre, como prevenir e tratar) e ingurgitamento mamário (definição, porque ocorre, qual a prevenção e seu manejo) (BRASIL, 2015).

A coleta dos dados deu-se por meio de 2 questionários estruturais visando a validação dos vídeos educativos inseridos no website YouTube® (Figura 2) e divulgados por meio do Facebook®, tendo se apropriado de Pasqualie Escala de Likert a partir de afirmações de acordo com os itens: Concordo Totalmente (CT), Concordo Parcialmente (CP) e Discordo (D). É uma escala de verificação, utilizada a fím de mensurar a concordância dos sujeitos a determinadas afirmações(JÚNIOR \& COSTA, 2014; DALMOLIN et al., 2016). 
Figura 2 - Vídeos educativos no website YouTube ${ }^{\circledast}$

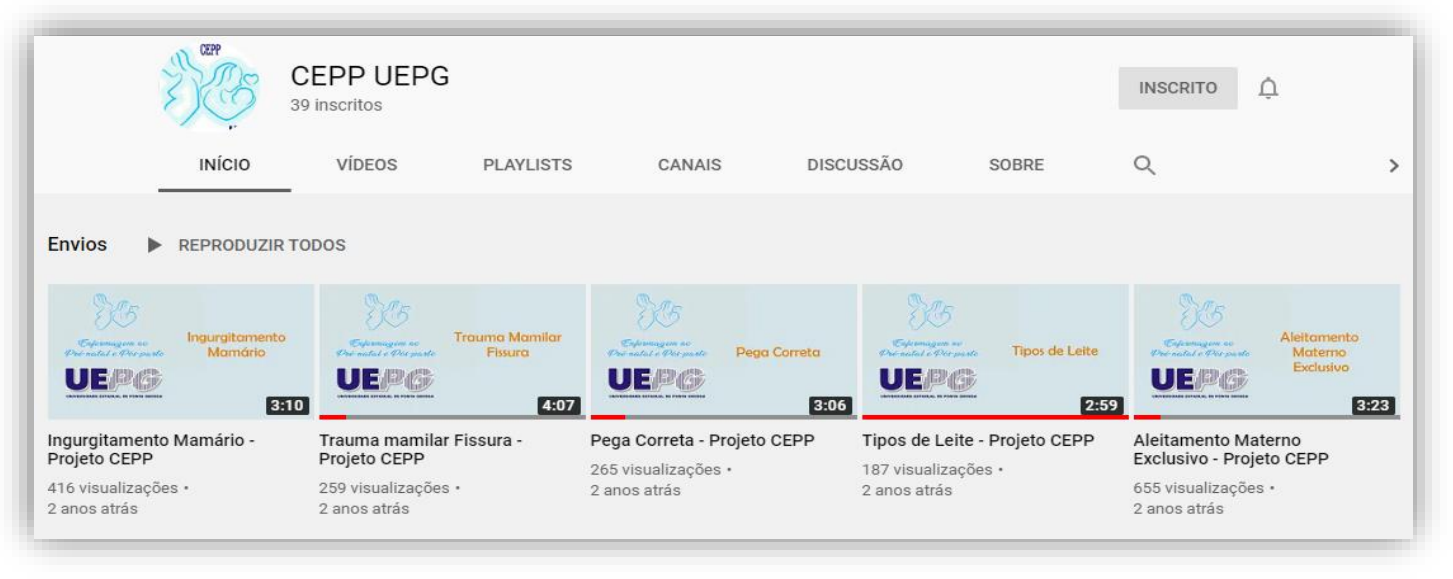

Fonte: autores (2018).

O questionário para as enfermeiras especialistas foi composto por 14 afirmativas, subdivididas nos seguintes critérios de Pasquali: Interatividade, Objetivos, Relevância/Eficácia e Clareza. Para os acadêmicos, o questionário foi composto de 17 afirmativas, subdivididas nos critérios: Objetivos, Acesso, Clareza, Estrutura/Apresentação, Relevância/Eficácia e Interatividade.

O estudo teve como critérios de inclusão: profissionais especialistas da área da saúde da mulher ou obstetrícia e acadêmicos do curso de Bacharelado em Enfermagem que estavam cursando a $1^{\mathrm{a}}, 2^{\mathrm{a}}$ e $3^{\mathrm{a}}$ série em 2018 e não possuíam aproximação com a disciplina de Saúde da Mulher que aborda o conteúdo de Aleitamento Materno. No entanto, os critérios de exclusão são os demais profissionais especialistas das diversas áreas do conhecimento e os demais acadêmicos do curso de Bacharelado em Enfermagem.

A análise dos dados foi desenvolvida pela estatística descritiva, a partir de frequência simples a partir dos critérios de Pasquali e conceitos da escala Likert. Todos os sujeitos foram convidados e assinaram o Termo de Consentimento Livre e Esclarecido de acordo com a resolução 466 de 2012. Esta pesquisa tem aprovação do Comitê de Ética em Pesquisa da Universidade Estadual de Ponta Grossa,sobparecer3.234.262 de 29 de março de 2019.

\section{RESULTADOS E DISCUSSÃO}

O questionário aplicado com as enfermeiras especialistas obteve os seguintes dados conforme critérios (Interatividade, Objetivos, Relevância/Eficácia e Clareza) e 
escala de Likert, numa visão técnica especializada do conteúdo e da ferramenta tecnológica, segundo o Quadro1.

Quadro1. Percentual das respostas de especialistas de acordo com critérios de Pasquali (1997) sobre instrumento de tecnologia educativa, Ponta Grossa, Paraná, Brasil, 2018. (N=4)

\begin{tabular}{|c|c|c|c|c|}
\hline CRITÉRIOS: & AFIRMATIVAS SOBRE OS VÍDEOS & CT & $\mathrm{CP}$ & $\mathrm{D}$ \\
\hline \multirow[t]{4}{*}{ INTERATIVIDADE } & $\begin{array}{l}\text { O conteúdo (AM) está adequado e o material } \\
\text { (vídeo) traz interatividade }\end{array}$ & $\begin{array}{l}100 \% \\
(4)\end{array}$ & 0 & 0 \\
\hline & $\begin{array}{c}\text { Oferece interação e envolvimento no processo } \\
\text { educativo }\end{array}$ & $\begin{array}{c}100 \% \\
(4)\end{array}$ & 0 & 0 \\
\hline & Possibilita o entendimento do conteúdo & $\begin{array}{l}100 \% \\
(4)\end{array}$ & 0 & 0 \\
\hline & $\begin{array}{l}\text { Fornece autonomia do usuário em relação à sua } \\
\text { operação }\end{array}$ & $\begin{array}{l}25 \% \\
(1)\end{array}$ & $\begin{array}{l}75 \% \\
(3)\end{array}$ & 0 \\
\hline \multirow[t]{4}{*}{ OBJETIVOS } & Estimula aprendizagem sobre conteúdo abordado & $\begin{array}{l}100 \% \\
(4)\end{array}$ & 0 & 0 \\
\hline & Estimula aprendizagem de novos conceitos & $\begin{array}{l}75 \% \\
(3)\end{array}$ & $\begin{array}{l}25 \% \\
(1)\end{array}$ & 0 \\
\hline & Permite buscar informações sem dificuldades & $\begin{array}{l}75 \% \\
(3)\end{array}$ & $\begin{array}{l}25 \% \\
(1)\end{array}$ & 0 \\
\hline & Possui estratégia de apresentação atrativa & $\begin{array}{l}100 \% \\
(4)\end{array}$ & 0 & 0 \\
\hline \multirow[t]{4}{*}{$\begin{array}{l}\text { RELEVÂNCIA } \\
\text { E EFICÁCIA }\end{array}$} & Disponibiliza fácil utilização e visualização & $\begin{array}{l}75 \% \\
(3)\end{array}$ & $\begin{array}{c}25 \% \\
(1)\end{array}$ & 0 \\
\hline & Desperta interesse ao visualizá-lo & $\begin{array}{l}100 \% \\
(4)\end{array}$ & 0 & 0 \\
\hline & Estimula mudança de comportamento & $\begin{array}{l}75 \% \\
(3)\end{array}$ & $\begin{array}{l}25 \% \\
(1)\end{array}$ & 0 \\
\hline & $\begin{array}{l}\text { Reproduz o conteúdo abordado em diferentes } \\
\text { contextos online }\end{array}$ & $\begin{array}{l}100 \% \\
(4)\end{array}$ & 0 & 0 \\
\hline \multirow[t]{2}{*}{ CLAREZA } & Apresenta informações de modo simples & $\begin{array}{c}100 \% \\
(4)\end{array}$ & 0 & 0 \\
\hline & Permite refletir sobre o conteúdo apresentado & $\begin{array}{l}100 \% \\
(4)\end{array}$ & 0 & 0 \\
\hline
\end{tabular}

Fonte:autores (2018).

Legenda: $\mathrm{AM}=$ aleitamento materno, $\mathrm{CT}=$ concordo totalmente, $\mathrm{CP}=$ concordo parcialmente e $\mathrm{D}=$ Discordo. Fonte: Dados da pesquisa mediante banco de dados do projeto CEPP. 
Destaca-se o critério Interatividade, subdividido em quatro questões afirmativas, das quais, três alcançaram 100\% de Concordância Total (CT), no qual somente uma afirmativa teve $75 \%$ de Concordância Parcial (CP), evidenciando a dúvida dos participantes em relação à autonomia do usuário em relação à operação do material educativo.

Os critérios subsequentes Objetivos, Relevância/Eficácia e Clareza obtiveram um resultado satisfatório, pois alcançaram $\geq 75 \%$ de CT. Destaca-se que na visão dos enfermeiros especialistas em Saúde da Mulher o material educativo atingiu seus objetivos, despertando o interesse do usuário ao transmitir a informação com clareza.

Já quanto à avaliação realizada com os alunos a partir dos critérios de Pasquali (Objetivos, Acesso, Clareza, Estrutura/Apresentação, Relevância/Eficácia e Interatividade), evidenciou que os mesmos possuíam uma visão desconhecida da temática aleitamento materno, pois não tiveram contato com a disciplina Saúde da Mulher, fazendo uma analogia com a comunidade, para a qual os vídeos são disponibilizados.

Sendo assim, todos os critérios foram considerados satisfatórios $(\geq 60 \%)$, com índice de concordância total o que evidencia que o material educativo proposto é uma ferramenta que auxilia no processo ensino-aprendizagem sobre a temática, conforme Quadro 2.

Quadro 2 - Percentual das respostas de acadêmicos do curso de Enfermagem de acordo com critérios de Pasquali (1997) sobre instrumento de tecnologia educativa, Ponta Grossa, Paraná,

Brasil, 2018. $(\mathrm{N}=38)$

\begin{tabular}{|c|c|c|c|c|}
\hline CRITÉRIOS & AFIRMATIVAS SOBRE OS VÍDEOS: & CT & CP & D \\
\hline OBJETIVOS & $\begin{array}{c}\text { Relaciona conteúdo abordado no seu dia a } \\
\text { dia }\end{array}$ & $\begin{array}{c}68,4 \% \\
(26)\end{array}$ & $\begin{array}{c}28,9 \% \\
(11)\end{array}$ & $\begin{array}{c}2,6 \% \\
(1)\end{array}$ \\
\hline & $\begin{array}{c}\text { Esclarece as dúvidas sobre o conteúdo } \\
\text { abordado }\end{array}$ & $\begin{array}{c}100 \% \\
(38)\end{array}$ & 0 & 0 \\
\hline & $\begin{array}{c}\text { Estimula aprendizagem sobre o conteúdo } \\
\text { abordado }\end{array}$ & $\begin{array}{c}84,2 \% \\
(32)\end{array}$ & $\begin{array}{c}10,5 \% \\
(4)\end{array}$ & $\begin{array}{c}2,6 \% \\
(1)\end{array}$ \\
\hline \hline & $\begin{array}{c}\text { Estimula aprendizagem de novos conceitos } \\
\text { ou fatos }\end{array}$ & $\begin{array}{c}81,6 \% \\
(31)\end{array}$ & $\begin{array}{c}18,4 \% \\
(7)\end{array}$ & 0 \\
\hline ACESSO & Permite-lhe buscar informações sem \\
dificuldades & $\begin{array}{c}81,6 \% \\
(31)\end{array}$ & $\begin{array}{c}18,4 \% \\
(7)\end{array}$ & 0 \\
\hline & Disponibiliza recursos adequados e & $63,2 \%$ & $34,2 \%$ & 0 \\
\hline
\end{tabular}




\begin{tabular}{|c|c|c|c|c|}
\hline & necessários para sua utilização & (24) & (13) & \\
\hline \multirow[t]{3}{*}{ CLAREZA } & $\begin{array}{l}\text { Apresenta informações necessárias para } \\
\text { melhor compreensão do conteúdo }\end{array}$ & $\begin{array}{c}97,4 \% \\
(37)\end{array}$ & $\begin{array}{c}2,6 \% \\
(1)\end{array}$ & 0 \\
\hline & Conteúdo da informação está adequado & $\begin{array}{c}100 \% \\
(38)\end{array}$ & 0 & 0 \\
\hline & Apresenta informações de modo simples & $\begin{array}{c}76,3 \% \\
(29)\end{array}$ & $\begin{array}{l}23,7 \% \\
(9)\end{array}$ & 0 \\
\hline \multirow[t]{2}{*}{$\begin{array}{l}\text { ESTRUTURA E } \\
\text { APRESENTAÇÃO }\end{array}$} & Apresenta conteúdo de forma organizada & $\begin{array}{c}100 \% \\
(38)\end{array}$ & 0 & 0 \\
\hline & Possui estratégia de apresentação atrativa & $\begin{array}{c}65,8 \% \\
(25)\end{array}$ & $\begin{array}{c}31,6 \% \\
(12)\end{array}$ & $\begin{array}{c}2,6 \% \\
(1)\end{array}$ \\
\hline \multirow[t]{4}{*}{$\begin{array}{l}\text { RELEVÂNCIA E } \\
\text { EFICÁCIA }\end{array}$} & $\begin{array}{l}\text { Permite-lhe refletir sobre o conteúdo } \\
\text { apresentado }\end{array}$ & $\begin{array}{c}84,2 \% \\
(32)\end{array}$ & $\begin{array}{l}15,8 \% \\
(6)\end{array}$ & 0 \\
\hline & Desperta seu interesse para utilizá-lo & $\begin{array}{c}71,1 \% \\
(27)\end{array}$ & $\begin{array}{c}28,9 \% \\
(11)\end{array}$ & 0 \\
\hline & $\begin{array}{c}\text { Estimula a mudança de comportamento em } \\
\text { você }\end{array}$ & $\begin{array}{c}71,1 \% \\
(27)\end{array}$ & $\begin{array}{c}26,3 \% \\
(10)\end{array}$ & $\begin{array}{l}2,6 \% \\
(1)\end{array}$ \\
\hline & $\begin{array}{l}\text { Reproduz o conteúdo abordado em } \\
\text { diferentes contextos }\end{array}$ & $\begin{array}{c}60,5 \% \\
(23)\end{array}$ & $\begin{array}{c}36,8 \% \\
(14)\end{array}$ & 0 \\
\hline \multirow[t]{2}{*}{ INTERATIVIDADE } & $\begin{array}{l}\text { Oferece interação, envolvimento ativo no } \\
\text { processo educativo }\end{array}$ & $\begin{array}{l}78,9 \% \\
(30)\end{array}$ & $\begin{array}{l}21,1 \% \\
(8)\end{array}$ & 0 \\
\hline & $\begin{array}{l}\text { Fornece autonomia do usuário em relação à } \\
\text { sua operação }\end{array}$ & $\begin{array}{l}78,9 \% \\
(30)\end{array}$ & $\begin{array}{l}21,1 \% \\
\quad(8)\end{array}$ & 0 \\
\hline TOTAL (n): & & $\begin{array}{c}100 \% \\
(38)\end{array}$ & $\begin{array}{c}100 \% \\
(38)\end{array}$ & $\begin{array}{c}100 \% \\
(38)\end{array}$ \\
\hline
\end{tabular}

Fonte:autores (2018)

Legenda: $\mathrm{CT}=$ concordo totalmente, $\mathrm{CP}=$ concordo parcialmente e $\mathrm{D}=$ Discordo. Fonte: Dados da pesquisa mediante banco de dados do projeto CEPP.

A educação em saúde pode apoiar-se em recursos tecnológicos, nas chamadas tecnologias de informação e comunicação, uma vez que contribuem no processo ensinoaprendizagem facilitando a comunicação e compreensão dos usuários. Assim, como ferramenta, o vídeo educativo pode ser utilizado, pois gera conhecimento, reflete na consciência crítica do espectador e permite a promoção de saúde(DALMOLIN et al., 2016).

As tecnologias utilizadas no processo educativo devem ser interativas, ou seja, devem promover envolvimento do usuário com a ferramenta tecnológica por meio de 
uma linguagem clara, possibilitando a interação e o processo do aprendizado (NUNES \& BEZERRA, 2015).

Logo, a interatividade é definida como a característica de uma interface digital, a qual possibilita trocas entre o usuário e a ferramenta (VALLE \& BOHADANA, 2012). Em relação à Interatividade do material educativo, 100\% (4) das enfermeiras acreditaram que o conteúdo abordado está adequado e que a ferramenta traz interatividade, concordando que o vídeo oferece interação e envolvimento no processo ensino-aprendizagem.

Em contrapartida, $75 \%$ (3) delas concordaram parcialmente que o material fornece autonomia do usuário em relação à sua operação, e apenas 25\% (1) concordaram totalmente, evidenciando a dúvida acerca da autonomia. Porém, segundo a literatura, a tecnologia interativa no processo educativo está interligada ao desenvolvimento da autonomia e pensamento crítico, ela gera uma crescente autonomia do usuário diante das informações que possuem acesso através da rede (OLIVEIRA, 1999; PEDRO \& CHACON, 2017).

A utilização de vídeos com fins educativos possibilita uma diferente exploração dos conteúdos abordados, por meio de uma melhor percepção das informações. Esta ferramenta gera a curiosidade e interesse pela busca de novas informações, contanto que seja utilizado de maneira adaptada aos objetivos do processo de aprendizagem (MOREIRA et al., 2013).

Nesta perspectiva, o recurso audiovisual pode ser utilizado como ferramenta facilitadora no processo ensino-aprendizagem, pois por meio dele, conquista-se a atenção do público, despertando seu interesse acerca da temática abordada (CAVALCANTI et al., 2017).

No que se refere à Clareza dos vídeos educativos as informações foram apresentadas de modo simples e a ferramenta permite refletir sobre o conteúdo apresentado. A combinação dos recursos áudio e visual permite uma maior assimilação da informação, o que gera facilidade na aprendizagem. Assim, o vídeo educativo proporciona uma maior capacidade de representação do que foi aprendido a partir do conhecimento adquirido por meio da tecnologia educativa (MOREIRA et al., 2013; QUADROS \& LOPEZ, 2014). 
Por sua vez, no Quadro2, quanto ao critério Objetivos, o material relaciona o conteúdo abordado no seu dia-a-dia e esclarece dúvidas sobre o conteúdo abordado, estimulando o processo de aprendizagem. Vale destacar que os vídeos educativos funcionam como ferramentas de incentivo a educação, pois o áudio e a imagem se associados podem aproximar os conteúdos conforme à realidade da população, sendo assim, despertam maior interesse e aprendizagem (LIMA et al., 2017).

Sobre o Acesso dos vídeos educativos, a literatura traz que o acesso à internet vem sendo progressivamente disponível ao alcance da população, atuando como um meio informativo e esclarecedor para os usuários que procuram informações relacionadas à saúde. Ainda, verifica-se uma ampliação do acesso à internet, bem como das mídias sociais, o que reflete no crescimento das informações transmitidas em larga escala e em um curto espaço de tempo (SANTOS et al., 2017).

Na visão dos acadêmicos, no critério Clareza, o material apresentou informações necessárias para melhor compreensão do conteúdo, pois o conteúdo da informação está adequado e as informações são apresentadas de modo simples. Nesse contexto, destacase a importância de que as tecnologias educativas e seus materiais sejam convenientes conforme o nível de escolaridade do público-alvo, pois a escolaridade tem influência direta na compreensão do conteúdo abordado (LIMA et al., 2017).

Por sua vez, no Critério Estrutura/Apresentação referentes aos vídeos educativos, os alunos acreditaram que o conteúdo foi apresentado de modo organizado e que possui estratégia de apresentação atrativa. De acordo com a literatura, os vídeos devem ser bem elaborados para que a aprendizagem se torne atrativa e eficaz ao objetivo proposto (CAVALCANTI et al., 2017; RAZERA et al., 2014).

Desta forma, destaca-se a necessidade da atratividade do vídeo educativo para a conquista de atenção do telespectador, para que o mesmo não perca o interesse. Para isso devem ser utilizados vários elementos, tais como imagens, texto e som no objeto promotor de conhecimento (DALMOLIN et al., 2016; ITAKUSSU et al., 2014).

Sendo assim, os vídeos educativos têm um reflexo positivo na promoção da educação e quando são corretamente desenvolvidos permitem uma reflexão e compreensão eficaz acerca da temática abordada. No entanto, para isso necessita de maior dedicação na estrutura e organização do material (RAZERA et al., 2014). 
Portanto, a interatividade deve ser priorizada, ela faz-se necessária para gerar um ambiente de diálogo com o espectador, atraindo-o para refletir sobre o tema abordado (CAVALCANTI et al., 2017).

Com relação à Interatividade, na opinião dos alunos, a ferramenta educativa ofereceu interação e envolvimento ativo no processo educativo e gera autonomia do usuário em relação à sua operação. Diversos recursos tecnológicos podem ser utilizados como ferramentas facilitadoras do processo ensino-aprendizagem, potencializando o empoderamento do usuário e a aprendizagem autônoma, sendo estas representadas por meio das TIC (DALMOLIN et al., 2016).

\section{CONCLUSÕES}

O estudo apropriou-se das TIC por acreditar serem ferramentas facilitadoras no processo ensino-aprendizagem. A estruturação dos vídeos educativos nesta pesquisa evidenciou que as tecnologias arraigadas à instrumentos facilitadores do processo de ensino-aprendizagem na educação em saúde acerca da temática, Aleitamento Materno, contribuiu ao surgimento da autonomia do usuário.

Acredita-se que a tecnologia educativa em forma de vídeo contribua para a atuação do profissional enfermeiro em suas ações educativas, junto à comunidade a partir da prática da educação em saúde. Destaca-se que na literatura há pouca produção relacionada as tecnologias de informação e comunicação na área da saúde e que outras pesquisas precisam ser realizadas a fim de evidenciar ainda mais a contribuição das tecnologias da comunicação e informação no processo de ensino-aprendizagem.

\section{AGRADECIMENTOS}

Agradecemos ao Nutead-UEPG pela criação e edição do material educativo proposto pelo trabalho, a Universidade Estadual de Ponta Grossa e ao Hospital Universitário dos Campos Gerais pela possibilidade de desenvolver a pesquisa e contribuir para a comunidade científica.

\section{REFERÊNCIAS}


ALVES, J.S.; OLIVEIRA, M.I.C. de; RITO, R.V.V.F. Orientações sobre amamentação na atenção básica de saúde e associação com o aleitamento materno exclusivo. Ciência \& Saúde Coletiva, v.23, n.4, p.1077-88, 2018. Disponível em: $<$ http://www.scielo.br/scielo.php?script=sci_abstract\&pid=S141381232018000401077\&lng=pt\&nrm=iso\&tlng=pt $>$.Acessoem 08, out. 2018.

AZEVEDO, A.R.R. et al. Clinical management of breastfeeding: knowledge of nurses. Escola Anna Nery,Rio de Janeiro v. 19, n.3, p.439-45, jul./set. 2015. Disponível em: $<$ http://www.scielo.br/scielo.php?pid=S141481452015000300439\&script=sci_arttext\&tlng=em>. Acesso em 08, out. 2018.

BAPTISTA, S. S., et al. Manejo clínico da amamentação: atuação do enfermeiro na Unidade de Terapia Intensiva Neonatal. Enfermagem da UFSM, v.5, n.1, p. 23-31, jan./mar.,2015. Disponível em:<https://periodicos.ufsm.br/reufsm/article/view/14687>. Acesso em 08, out. 2018.

BRASIL. Ministério da Saúde. Saúde da criança: aleitamento materno e alimentação complementar. Brasília, DF, 2015. p.84.

CAVAlCANTI, R.J. Construção De Vídeo Educativo Para a Promoção Da Saúde Ocular Em Escolares. Texto Contexto Enfermagem, Florianópolis, v.26, n.2, p.1-11, jul. 2017.

Disponível em:<http://www.scielo.br/scielo.php?script=sci_arttext\&pid=S0104-07072017 000200334\&lng=en\&tlng=en>. Acesso em 08, out. 2018.

DALMOLIN A., et al. Vídeo educativo como recurso para educação em saúde a pessoas com colostomia e familiares. Revista Gaúcha de Enfermagem Online, v.37, n.(spe: e68373), p.1-9, abr. 2016. Disponível em: http://www.scielo.br/scielo.php?pid=S1983-14472016000500408\&script=sci_abstract $>$. Acesso em 08, out. 2018.

ITAKUSSU, E.Y., et al. Elaboração de vídeo educativo sobre uso da malha compressiva após queimadura. Revista Brasileira de Queimaduras, v.13, n.4, p. 2369, 2014. Disponível em: $<\quad$ http://www.rbqueimaduras.com.br/exportpdf/225/v13n4a05.pdf>. Acessoem 08, out. 2018.

JÚNIOR, S.D. da S.; \& COSTA, F.J. Measurement and Verification Scales: a Comparative Analysis between the Likert and Phrase Completion Scales. PMKT - 
Brazilian Journal of Marketing, Opinion, and Media Research, São Paulo, v. 15, p. 1-15, Out., $2014 . \quad$ Disponívelem:< http://www.revistapmkt.com.br/Portals/9/Volumes/15/en-GB/1_Measurement\%20and $\% 20$ Verification $\% 20$ Scales $\% 20 \mathrm{a} \% 20$ Comparative $\% 20$ Analysis $\% 20$ between $\% 20$ the $\% 2$ 0Likert\%20and\%20Phrase\%20Completion\%20Scales.pdf. Acessoem 08, out. 2018.

LIMA, M.B. de, et al. Construção e validação de vídeo educativo para orientação de pais de crianças em cateterismo intermitente limpo. Escola de Enfermagem da USP, São Paulo, v.51, n.1, p.1-7, dez. 2017. Disponível em: $<$ http://www.scielo.br/scielo.php?script=sci_arttext\&pid=S0080$62342017000100462 \& \operatorname{lng}=\mathrm{p}$ t\&tlng=pt>. Acesso em 08, out. 2018.

MARTINS, M. Capacitação a distância dos profissionais em saúde em aleitamento materno[monografia]. Curitiba: Educação a Distância, Universidade Federal do Paraná; 2013.

MOREIRA, C.B., et al. Construção de um Vídeo Educativo sobre Detecção Precoce do Câncer de Mama. Revista Brasileira de Cancerologia, v.59, n.3, p. 401-7, mai. 2013. Disponível em:<http://www1.inca.gov.br/rbc/n_59/v03/pdf/10-artigoconstrucao-video-educativo-sobre-deteccao-precoce-cancer-mama.pdf $>$. Acesso em 08, out. 2018.

NUNES, C.H.F.; BEZERRA A.C.R. Fundamentos da interação no contexto da educação a distância: uma análise dos processos interativos na faculdade Estácio Polo Arapiraca. In: Anais $10^{\circ}$ Encontro Internacional de Formação de Professores e $11^{\circ}$ Fórum Permanente de Inovação Educacional; 2017, mai 15-19; Aracaju, Sergipe, Brasil. Aracaju: Universidade Tiradentes - Sergipe; 2015. p. 1-17.

OLIVEIRA, M.R.N.S. Tecnologias interativas e Educação. Educação em debate,Fortaleza, n.37, $\quad$ p. 150-6, $1999 . \quad$ Disponível em:<http://www.repositorio.ufc.br/bitstream/riufc/14332/3/1999_art_mrnoliveira.pdf>. Acesso em 08, out. 2018.

PEDRO, K.M.; CHACON, M.C.M. Competências Digitais e Superdotação: uma Análise Comparativa sobre a Utilização de Tecnologias. Revista Brasileira Educação $\begin{array}{lllll}\text { Especial, } & \text { v.23, } & \text { n.4, } & \text { p. } & \text { 517-30, }\end{array}$ 
em: $<$ http://www.scielo.br/scielo.php?script=sci_abstract\&pid=S1413-

65382017000400517\&lng =pt\&nrm=iso\&tlng=pt >. Acesso em 08, out. 2018.

QUADROS, M.; LOPEZ, D.C. As redes sociais como ferramentas de interatividade no radiojornalismo: uma proposta metodológica. Conexão, Comunicação e Cultura, v.13, n.26, p. 37-52, jul./dez. 2014. Disponível em: <http://www.ucs.br/etc/revistas/index.php/conexao/article/view/2725/1758>. Acesso em 08, out. 2018.

RAZERA, A.P.R., et al. Vídeo educativo: estratégia de ensino-aprendizagem para pacientes em tratamento quimioterápico. Ciência, Cuidado e Saúde, v.13, n.1, p. 1738, jan./mar. $2014 . \quad$ Disponível em:<http://www.periodicos.uem.br/ojs/index.php/CiencCuidSaude/article/view/19659/p df_156>. Acesso em 08, out. 2018.

SANTOS, G.S., et al. Reflexões sobre o uso das redes sociais virtuais no cuidado às pessoas com doença crônica. Revista de Enfermagem UFPE, Recife, v.11, n.2, p. 724-30, fev. $2017 . \quad$ Disponível em:<https://periodicos.ufpe.br/revistas/revistaenfermagem/article/download/11992/145 58>. Acesso em 08, out. 2018.

SANTOS, M.S.M. TICS com jogos educacionais na educação inclusiva para alunos com necessidade educacional especial em deficiência intelectual [Artigo de Conclusão de Curso]. Sant'Ana do Livramento: Especialização em Mídias de Educação, Universidade Federal de Santa Maria; 2017.

SILVA, A.C., et al. Tecnologias em aleitamento materno: revisão integrativa. Revista Brasileira de Promoção a Saúde, Fortaleza, v.29, n.3, p. 439-46, jul./set. 2016. Disponível em:< http://periodicos.unifor.br/RBPS/article/view/4812/pdf >. Acesso em 08, out. 2018.

SILVA, D.S.S., et al. Promoção do aleitamento materno: políticas públicas e atuação do enfermeiro. Cadernos UniFOA,Volta Redonda,v. 12, n.35, p. 135-40, dez. 2017.

Disponível em:<http://revistas.unifoa.edu.br/index.php/cadernos/article/view/483/1286>. Acesso em 08, out. 2018. 
VALLE, L. do; BOHADANA E.D.B. Interação e interatividade: por uma reantropolização da EaD online. Educação e Sociedade, Campinas v.33, n.121, p.97384 , out./dez. 2012.

Disponível em: $<$ http://www.scielo.br/scielo.php?script=sci_arttext\&pid=S010173302012000400003>. Acesso em 08, out. 2018.

VENANCIO, S.I., et al. Associação entre o grau de implantação da Rede Amamenta Brasil e indicadores de amamentação. Cadernos de Saúde Pública, v.32, n.3, p. 1-10, mar. 2016. Disponível em:<http://www.scielo.br/scielo.php?pid=S0102311X2016000300704\&script=sci _abstract\&tlng=pt>. Acesso em 08, out. 2018.

Recebido em: 15 de outubro de 2019. Aceito em: 04 de março de 2020. 